\title{
Case report of amiodarone-associated allergic pneumonitis amidst the COVID-19 pandemic
}

\author{
Meor Azraai ${ }^{1, * \mathbb{D}}$, Marcus McMahon ${ }^{2}$, Ronald Dick $^{1}$ \\ ${ }^{1}$ Department of Cardiology, Epworth Healthcare, Melbourne, 3121 Victoria, Australia \\ ${ }^{2}$ Department of Respiratory Medicine, Epworth Healthcare, Melbourne, 3121 Victoria, Australia \\ *Correspondence: meor.azraaimeorahmad@epworth.org.au (Meor Azraai)
}

DOI:10.31083/j.rcm.2021.01.267

This is an open access article under the CC BY 4.0 license (https://creativecommons.org/licenses/by/4.0/).

Submitted: 29 November 2020 Revised: 28 December 2020 Accepted: 29 December 2020 Published: 30 March 2021

Amiodarone is a common antiarrhythmic drug that is utilised in clinical practice and is associated with pulmonary toxicity. The most common form of pulmonary complication is interstitial pneumonitis which is treated with discontinuation of amiodarone and initiation of corticosteroids. Amiodarone-induced pulmonary eosinophilia is a rare complication of amiodarone therapy, with blood and pulmonary eosinophilia the predominant features. During the COVID-19 era, the incidence of delay in treatment of pulmonary pathology is also delayed due to the effort of excluding COVID-19 infection. Here we report a case of a 64-year-old man who developed eosinophilic pneumonia after initiation of amiodarone therapy, and the investigations required to exclude other forms of pulmonary toxicity. We also reviewed the effect of COVID-19 testing in the management of patients presenting with respiratory distress.

\section{Keywords}

Amiodarone; Pulmonary eosinophilia; COVID-19; Amiodarone-induced pulmonary toxicity; Corticosteroids

\section{Introduction}

Amiodarone is the most commonly used antiarrhythmic drug to treat or prevent recurrences of supraventricular and ventricular arrhythmias [1]. Amiodarone is associated with adverse effect that can affect multiple organs, including pulmonary toxicity. There are multiple manifestations of amiodarone-induced pulmonary toxicity ranging from interstitial pneumonitis (most common) to acute respiratory distress syndrome, and amiodarone-induced eosinophilic pneumonitis is a rare complication of pulmonary toxicity [2].

Furthermore, the incidence of misdiagnosis or late institution of treatment has increased during the era of COVID-19. Here we describe a case of amiodarone-induced eosinophilic pneumonitis that present as sub-acute respiratory failure and the effect of COVID-19 testing in delaying diagnosis and management.

\section{Case report}

A 64-year-old man was admitted to hospital in August 2020 for an elective mitral valve replacement secondary to symptomatic functional mitral valve regurgitation. His past medical history includes a previous mechanical aortic valve replacement secondary to a bicuspid aortic valve stenosis and hypertension. A pre-surgery coronary angiography revealed no coronary artery disease and COVID-19 PCR nasopharyngeal swab was negative. During his third day post-operation, he developed an episode of atrial fibrillation with rapid ventricular rate in intensive care unit. Intravenous amiodarone of $1200 \mathrm{mg}$ in 24 hours was initiated and later changed to oral amiodarone $200 \mathrm{mg}$ three times a day after the patient converted to sinus rhythm.

On day 15 of his inpatient stay, he developed worsening shortness of breath, productive cough and worsening hypoxia. On examination, he was hypoxic with an oxygen saturation of $89 \%$ on room air with bilateral crackles up to the mid-zone of the lungs on auscultation and he was afebrile. His arterial blood gas results were as follows: $\mathrm{pH}-7.48, \mathrm{p} 0^{2}-$ $76 \mathrm{mmHg}, \mathrm{pCO}_{2}-35 \mathrm{mmHg}$ and bicarbonate $-23 \mathrm{mmol} / \mathrm{L}$. Other laboratory findings were an elevated white cell count of $20 \times 10^{9} / \mathrm{L}$ with an eosinophilia of $1.6 \times 10^{9} / \mathrm{L}$ and a C-reactive protein level of $120 \mathrm{mg} / \mathrm{L}$. His chest $\mathrm{x}$-ray revealed confluent perihilar and bilateral lung base consolidation consistent with pulmonary oedema (Fig. 1a). Further investigations with transthoracic echocardiography confirmed a normal ejection fraction with good function of the mitral valve prosthesis. A high-resolution CT scan of the chest revealed extensive ground glass opacity in both lungs, sparing the apical region with areas of patchy consolidation (Fig. 2). Furosemide and piperacillin/tazobactam were started and he was transferred to the intensive care unit where he was subsequently intubated due to respiratory fatigue. A repeat COVID-19 PCR from a swab and sputum sample were negative. His bronchoalveolar lavage (BAL) revealed a white cell count of $350 \mu \mathrm{L}$ with $80 \%$ eosinophils. Bacterial and viral culture were negative.

After a delay in the initiation of prednisolone, he was started on $1 \mathrm{~g}$ of methylprednisolone and amiodarone was stopped on day 20 of his inpatient stay. He had good response to treatment with normalisation of eosinophil count and was subsequently extubated after five days of treatment with methylprednisolone, which was then stopped. On day 


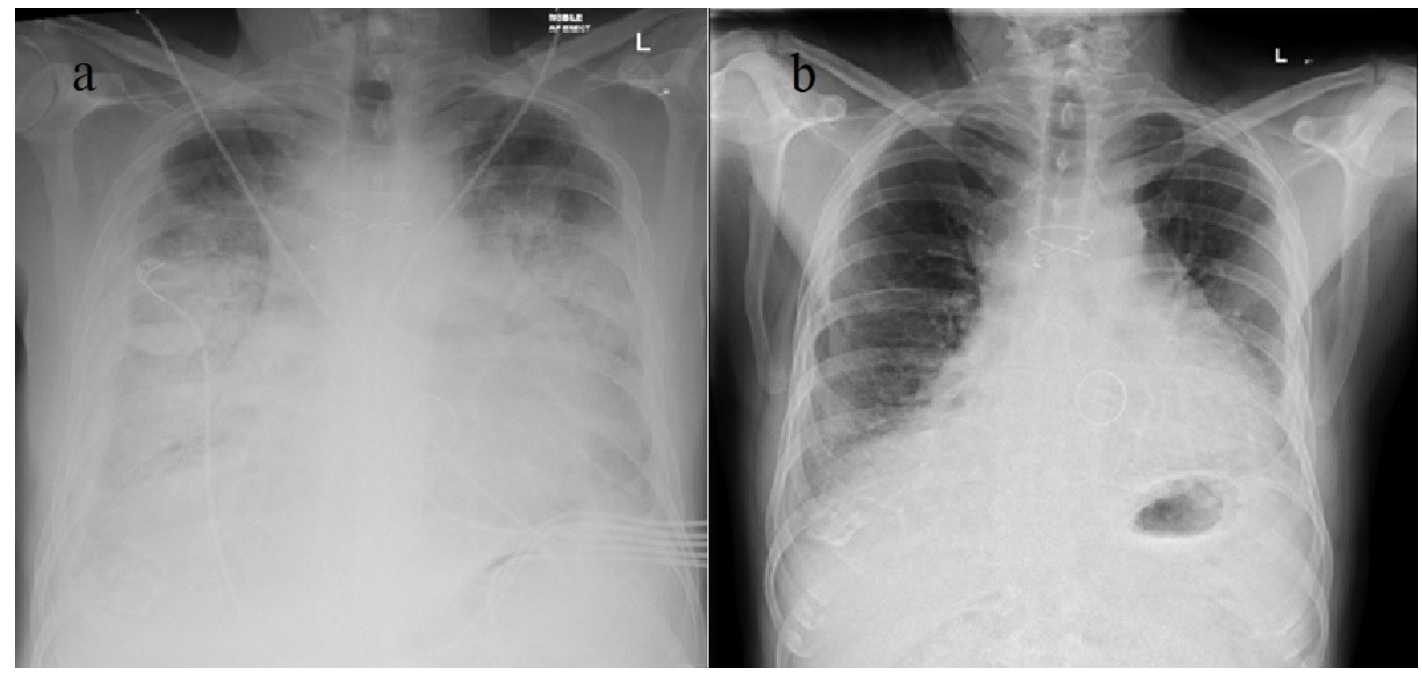

Fig. 1. (a) New onset of diffuse perihilar and lung infiltrates on initial chest $x$-ray, which was initially thought to represent pulmonary oedema figure 1. (b) Complete resolution of lung infiltrates on repeat chest $x$-ray on day 60 of admission. The patient was clinically better without any oxygen supplementation required.

30 of his inpatient stay, he developed another episode of type 1 respiratory failure with a chest $\mathrm{x}$-ray showing bilateral infiltrates throughout both mid and lower zone and recurrence in eosinophilia count to $1.9 \times 10^{9} / \mathrm{L}$. COVID-19 PCR on both nasopharyngeal swab and sputum sample were negative. He was then started on $50 \mathrm{mg}$ of prednisolone for 6 weeks and recovered with resolution of chest $\mathrm{x}$-ray changes (Fig. 1b) and normalisation of eosinophil count. The patient was discharged back home and is now clinically stable off prednisolone and amiodarone.

\section{Discussion}

The incidence of amiodarone-induced pulmonary toxicity ranges between $5-13 \%$ [3]. The most common presentation is interstitial pneumonitis which commonly presents after treatment with amiodarone for an average period of 2 months. The symptoms of Amiodarone pulmonary toxicity range from dyspnoea, non-productive cough and fever. Chest imaging usually shows bilateral patchy interstitial or alveolar infiltrates [4] in amiodarone pulmonary toxicity. Mainstay of treatment are discontinuation of amiodarone and initiating corticosteroids [5].

Amiodarone-induced pulmonary eosinophilia is a rare manifestation of amiodarone-induced pulmonary toxicity. The presentation ranges from acute to chronic form, with the acute form typically presenting less than one month after the initiation of amiodarone. Patients typically present with fever, non-productive cough and dyspnoea [6]. Diagnostic criteria include $>1 \times 10^{9}$ eosinophils/L in peripheral blood, $\geq 25 \%$ eosinophils on BAL cell count, and/or demonstration of lung tissue eosinophilia on lung biopsy [7]. The diagnosis also requires careful review of the medication history and exclusion of other possible aetiologies, such as infections or toxins.

Amiodarone-induced eosinophilic pneumonitis is most frequently diagnosed using lung biopsy. A study by Larsen et al. diagnosed four cases of eosinophilic pneumonitis with pulmonary biopsy in a retrospective study of 75 patients with suspected amiodarone-induced pulmonary toxicity [8]. However, the diagnosis of amiodarone-induced pulmonary eosinophilia using peripheral or BAL eosinophilia are limited. Currently, only three case reports of amiodaroneinduced eosinophilic pneumonitis diagnosed by peripheral eosinophilia has been published, with two of them demonstrating $20 \%$ and $80 \%$ eosinophils on BAL respectively [9$11]$.

Both blood and/or BAL eosinophilia have been detected in patients with other manifestation of amiodarone-induced pulmonary toxicity [6]. Coudert et al. studied the significance of BAL findings in fifteen patients with amiodarone pneumonitis. The study showed that the average eosinophilia percentage was $6 \%$ and the highest was $16 \%$, but none fulfilled the criteria for a diagnosis of eosinophilic pneumonitis [8].

Question remains regarding the pathophysiology of amiodarone-induced pulmonary eosinophilia. Possible explanation includes direct cytotoxicity to type II pneumocytes, T-helper lymphocytes predominant inflammation and activation of the angiotensin enzyme system [8]. More research is needed to investigate the correlation between the effects of amiodarone on pneumocytes and activation of eosinophils.

Although a histopathology examination of a lung biopsy is an excellent way to confirm diagnosis, there is a strong temporal relationship between the initiation of amiodarone, distinctive radiological changes, blood eosinophilia, and presence of eosinophils on BAL. This is further confirmed by recurrence of symptoms, radiological changes, and blood eosinophilia with early cessation of corticosteroids. Therefore, there was no need for a lung biopsy to confirm diagnosed amiodarone pneumonitis. 

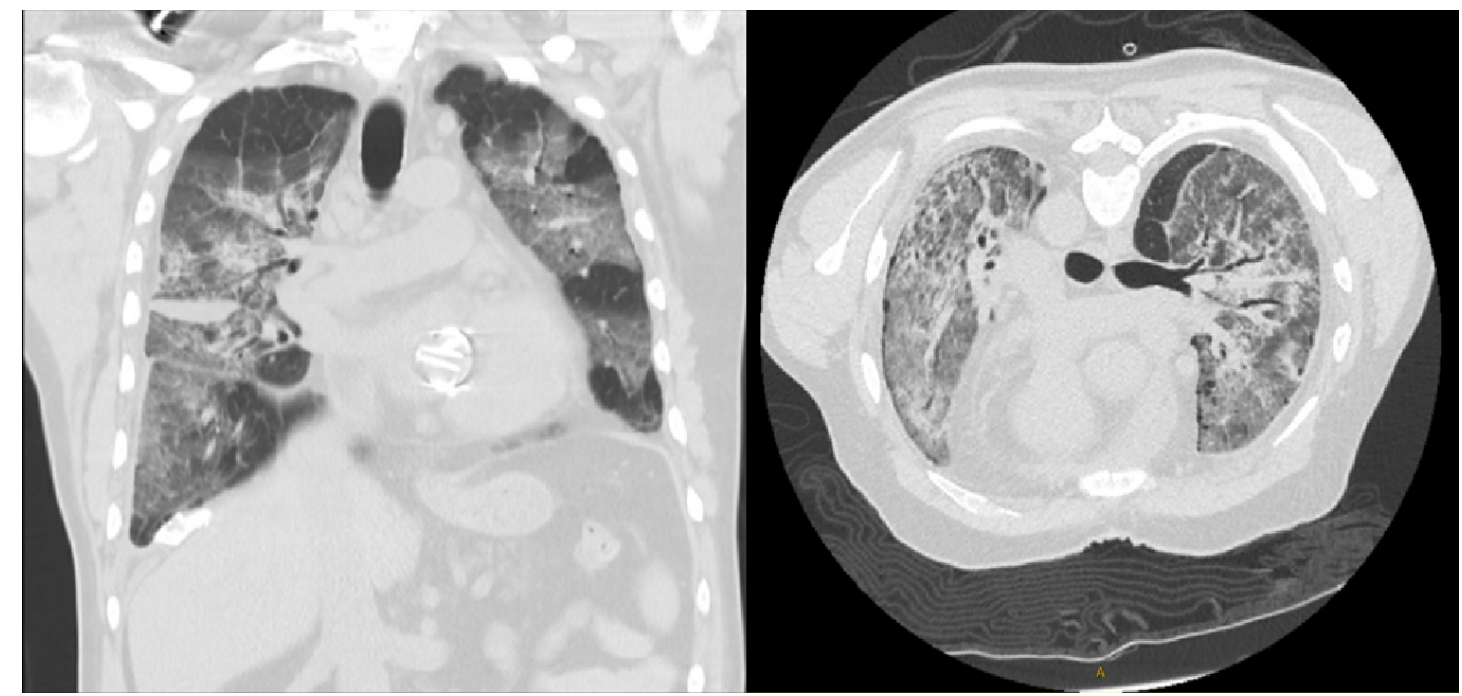

Fig. 2. High resolution CT chest 12 days after administration of amiodarone. Extensive bilateral ground glass opacity with areas of patchy consolidation are seen. The differential diagnosis of this chest imagine are acute pulmonary oedema, bilateral bacterial pneumoniae, viral pneumonitis and interstitial pneumonitis.

Furthermore, the patient had repeated COVID-19 PCR testing due to the development of respiratory distress and bilateral ground glass opacity on radiology. This has caused delay in reaching the final diagnosis and institution of steroids.

There are other cases of delayed diagnosis of reversible life-threatening condition due to COVID-19 such as myocardial infarction [12] or Kawasaki disease [13]. In both cases, important investigations and timely administration of lifesaving treatments were delayed. Before the COVID-19 era, these patients would likely have been referred immediately for further evaluation.

Strategies should be implemented to minimize diagnostic errors. This ranges from optimizing workflow, using people-focused interventions to implementing organizational strategies [14]. Open communication between clinicians should be encouraged and practicing 'diagnostic huddles' where cases are discussed to determine whether anything has been missed. Clear policies from the state and institution will assist in reducing diagnostic error [15]. Standardized metrics should be developed to monitor diagnostic performances and outcomes and evaluate how COVID-19 diagnostic error can be reduced [16]. The patient's case was discussed at a multi-disciplinary team (MDT) meeting, which includes multiple specialties, after he was discharged. The meeting highlighted the need for a high index of suspicion of iatrogenic cause of respiratory failure during the COVID-19 pandemic and improved communication between specialties and the use of 'diagnostic huddles' to reduce the risk of diagnostic errors.

\section{Conclusions}

This case emphasizes on the importance of recognising eosinophilic pneumoniae as a form of amiodarone induced pulmonary toxicity and it should be suspected in patients pre- senting with respiratory symptoms and radiographic findings after exclusion of other aetiologies.

\section{Author contributions}

MA: Data collection, literature review, write up of manuscript; MMM: Contributed to interpretation of results and review of manuscript; RD: Contributed to interpretation of results and review of manuscript.

\section{Ethics approval and consent to participate}

This study was consented by the patient and approved by the Ethics Committee of the Epworth Healthcare, Melbourne, Australia.

\section{Acknowledgment}

There are no acknowledgments to disclose.

\section{Funding}

This research received no external funding.

\section{Conflict of interest}

The author declares no conflicts of interest.

\section{References}

[1] Latini R, Tognoni G, Kates RE. Clinical pharmacokinetics of amiodarone. Clinical Pharmacokinetics. 1984; 9: 136-156.

[2] Dusman RE, Stanton MS, Miles WM, Klein LS, Zipes DP, Fineberg NS, et al. Clinical features of amiodarone-induced pulmonary toxicity. Circulation. 1990; 82: 51-59.

[3] Camus P, Colby TV, Rosenow EC. Amiodarone pulmonary toxicity. Drug-Induced and Iatrogenic Respiratory Disease (pp. 244263). 2010.

[4] Wolkove N, Baltzan M. Amiodarone pulmonary toxicity. Canadian Respiratory Journal. 2009; 16: 43-48. 
[5] Schwaiblmair M, Berghaus T, Haeckel $\mathrm{T}$, Wagner $\mathrm{T}$, von Scheidt W. Amiodarone-induced pulmonary toxicity: an underrecognized and severe adverse effect? Clinical Research in Cardiology. 2010; 99: 693-700.

[6] Papiris SA, Triantafillidou C, Kolilekas L, Markoulaki D, Manali ED. Amiodarone. Drug Safety. 2010; 33: 539-558.

[7] Cottin V, Frognier R, Monnot H, Levy A, DeVuyst P, Cordier J. Chronic eosinophilic pneumonia after radiation therapy for breast cancer. European Respiratory Journal. 2004; 23: 9-13.

[8] Larsen BT, Vaszar LT, Colby TV, Tazelaar HD. Lymphoid hyperplasia and eosinophilic pneumonia as histologic manifestations of amiodarone-induced lung toxicity. American Journal of Surgical Pathology. 2012; 36: 509-516.

[9] LeVee A, Trieu M, Bhattacharyya S, Sandhu G. Eosinophilic pneumonia: a rare manifestation of amiodarone toxicity diagnosed using traditional bronchoscopy. Respiratory Medicine Case Reports. 2019; 27: 100856

[10] Lavaud F, Cossart C, Tack V, Bonnaud G. Follow-up of blood and alveolar hyper-eosinophilia in pneumopathy induced by amiodarone. Therapie. 1990; 45: 49-51. (In French)

[11] Alqaid A, Baskaran G, Dougherty C. Amiodarone-induced lung injury with bilateral lung pneumonitis and peripheral eosinophilia. American Journal of Therapeutics. 2016; 23: e1121e1123.

[12] Yousefzai R, Bhimaraj A. Misdiagnosis in the COVID-19 era: when zebras are everywhere, don't forget the horses. JACC: Case Reports. 2020; 2: 1614-1619.

[13] Harahsheh AS, Dahdah N, Newburger JW, Portman MA, Piram $\mathrm{M}$, Tulloh R, et al. Missed or delayed diagnosis of Kawasaki disease during the 2019 novel coronavirus disease (COVID-19) pandemic. Journal of Pediatrics. 2020; 222: 261-262.

[14] Gandhi TK, Singh H. Reducing the risk of diagnostic error in the COVID-19 era. Journal of Hospital Medicine. 2020; 15: 363-366.

[15] Mossa-Basha M, Medverd J, Linnau KF, Lynch JB, Wener MH, Kicska G, et al. Policies and guidelines for COVID-19 preparedness: experiences from the University of Washington. Radiology. 2020; 296: E26-E31.

[16] Grange ES, Neil EJ, Stoffel M, Singh AP, Tseng E, Resco-Summers $\mathrm{K}$, et al. Responding to COVID-19: the UW Medicine Information Technology Services Experience. Applied Clinical Informatics. 2020; 11: 265-275. 